\title{
Lentivirus-mediated interleukin-1 $\beta$ (IL-1 $\beta$ ) knock-down in the hippocampus alleviates lipopolysaccharide (LPS)-induced memory deficits and anxiety- and depression-like behaviors in mice
}

Mengmeng $\mathrm{Li}^{1,2,3 \dagger}$, Chenli $\mathrm{Li}^{1,2,3 \dagger}$, Hanjie Yu $\mathrm{u}^{1,2,3 \dagger}$, Xiongxiong Cai ${ }^{1,2,3}$, Xinbei Shen ${ }^{1,2,3}$, Xin Sun ${ }^{1,2,3}$, Jinting Wang ${ }^{1,2,3}$, Yanhua Zhang ${ }^{1,2,3}$ and Chuang Wang ${ }^{1,2,3,4^{*}}$ (D)

\begin{abstract}
Background: Recent evidence has suggested that peripheral inflammatory responses induced by lipopolysaccharides (LPS) play an important role in neuropsychiatric dysfunction in rodents. Interleukin-1 $\beta$ (IL-1 $\beta$ ), a pro-inflammatory cytokine, has been proposed to be a key mediator in a variety of behavioral dysfunction induced by LPS in mice. Thus, inhibition of IL-1 $\beta$ may have a therapeutic benefit in the treatment of neuropsychiatric disorders. However, the precise underlying mechanism of knock-down of IL-1 $\beta$ in repairing behavioral changes by LPS remains unclear.

Methods: The mice were treated with either IL-1 $\beta$ shRNA lentivirus or non-silencing shRNA control (NS shRNA) lentivirus by microinjection into the dentate gyrus (DG) regions of the hippocampus. After 7 days of recovery, LPS $(1 \mathrm{mg} / \mathrm{kg}$, i.p.) or saline was administered. The behavioral task for memory deficits was conducted in mice by the novel object recognition test (NORT), the anxiety-like behaviors were evaluated by the elevated zero maze (EZM), and the depression-like behaviors were examined by the sucrose preference test (SPT) and the forced swimming test (FST). Furthermore, the levels of malondialdehyde (MDA), superoxide dismutase (SOD), nuclear factor erythroid-derived 2-like 2 (Nrf2), heme oxygenase 1 (HO1), IL-1ß, tumor necrosis factor (TNF-a), neuropeptide VGF (non-acronymic), and brainderived neurotrophic factor (BDNF) were assayed.

Results: Our results demonstrated that IL-1 $\beta$ knock-down in the hippocampus significantly attenuated the memory deficits and anxiety- and depression-like behaviors induced by LPS in mice. In addition, IL-1 $1 \beta$ knock-down ameliorated the oxidative and neuroinflammatory responses and abolished the downregulation of VGF and BDNF induced by LPS.

Conclusions: Collectively, our findings suggest that IL-1 $\beta$ is necessary for the oxidative and neuroinflammatory responses produced by LPS and offers a novel drug target in the IL-1//oxidative/neuroinflammatory/neurotrophic pathway for treating neuropsychiatric disorders that are closely associated with neuroinflammation, oxidative stress, and the downregulation of VGF and BDNF.
\end{abstract}

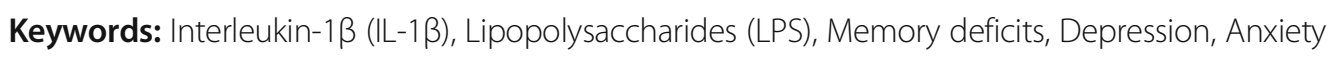

\footnotetext{
* Correspondence: wanglovechuang@163.com; wangchuang@nbu.edu.cn

${ }^{\dagger}$ Equal contributors

'Ningbo Key Laboratory of Behavioral Neuroscience, Ningbo University

School of Medicine, 818 Fenghua Road, Ningbo, Zhejiang 315211, People's

Republic of China

${ }^{2}$ Zhejiang Provincial Key Laboratory of Pathophysiology, Ningbo University

School of Medicine, 818 Fenghua Road, Ningbo, Zhejiang 315211, People's

Republic of China

Full list of author information is available at the end of the article
} 


\section{Background}

Growing evidence suggests a close link between neuroinflammation and neuropsychiatric disorders (e.g., memory deficits, depression, and anxiety) [1-4]. Acute treatment with the cytokine inducer lipopolysaccharide (LPS) is widely accepted in animal models to investigate the relationship between neuroinflammation and memory deficit, anxiety, and depression [2, 5, 6]. Indeed, behavioral dysfunction can be observed in LPS-stimulated animal models together with elevated pro-inflammatory cytokine levels and oxidative stress parameters in the hippocampus and other brain regions [7-10]. There is growing evidence that blocking the interleukin-1 $\beta$ (IL-1 $\beta$ ) receptor is a therapeutic strategy for neuropsychiatric behavioral dysfunction in rodents $[11,12]$, indicating IL- $1 \beta$ as a key candidate for the treatment of neuropsychiatric disorders. IL-1 $\beta$ administration induces fatigue, anhedonia, weight loss, impaired social interaction, and memory deficits in animal models [13-15]. Therefore, insight into the factors affecting IL- $1 \beta$ may lead to novel strategies for establishing a treatment for neuropsychiatric disorders. However, the role of IL-1 $\beta$ in LPSinduced behavioral dysfunction and neurotrophic factor loss [7-10] has not been elucidated in mice. We evaluated whether IL-1 $\beta$ knock-down induced by lentivirus in the hippocampus shows memory enhancing, anti-depression, and anxiolytic effects in the background of acute neuroinflammation induced by LPS in mice.

Increasing evidence indicates that the neurotrophic hypothesis of neuropsychiatric disorders is based on the downregulation of brain-derived neurotrophic factor (BDNF) and the neuropeptide VGF (no acronym) expression in the brain [16-21]. In addition, previous studies have demonstrated that LPS-induced pro-inflammatory cytokine mediate the generation of oxidative parameters $[9,22]$ and the impairment of BDNF [23] and VGF functions [7]. Intervening in the process of neuroinflammation along with oxidative stress may be beneficial for the therapy of neuropsychiatric disorders. However, very few studies have assessed the relationship between IL-1 $\beta$-mediated neuroinflammatory/oxidative signaling and BDNF or VGF in neuropsychiatric disorders. In our present work, we further assessed whether the possible protective effects of this IL-1 $\beta$ knock-down are associated with a decrease in pro-inflammatory cytokines and oxidative parameters, which are further implicated in the changes of BDNF and VGF in LPS-challenged mice.

\section{Methods}

\section{Animals}

Experiments were conducted on 8- to 10-week-old male imprinting control region (ICR) mice born and reared in the Zhejiang Academy of Medical Sciences, China. All animals were maintained at $22 \pm 3{ }^{\circ} \mathrm{C}$ and $60 \% \pm 5 \%$ relative humidity under a 12-h light/dark cycle (lights on at 7:00 a.m.) with ad libitum access to food and water. All animal experiments were conducted on animals outside of their housing area in a separate procedure room. All animal experiments were performed according to the National Institutes of Health (NIH) Guide for the Care and Use of Laboratory Animals (NIH Publication No. 80-23, revised 1996) and were approved by the Institutional Animal Care and Use Committee of the Medical School of Ningbo University.

In the present study, animals were divided into four experimental groups ( $n=16$ mice/group) for behavioral and biochemical assessments. Subsequent treatments were as follows: (1) Group 1 was treated with the nonsilencing shRNA control (NS shRNA) lentivirus and saline. (2) Group 3 was treated with the IL-1 $\beta$ shRNA lentivirus and saline. (3) Group 2 was treated with the NS shRNA lentivirus and LPS (1.0 mg/kg, i.p.). (4) Group 4 was treated with the IL- $1 \beta$ shRNA lentivirus and LPS $(1.0 \mathrm{mg} / \mathrm{kg}$, i.p.). All behavioral testing were performed during the light period (between 8:00 and 17:00) under dim light. At the end of all treatments and behavioral tests, the mice were killed and the bilateral hippocampus (32 hippocampi per group) was removed for biochemical analyses.

\section{Construction of the IL-1 $\beta$ shRNA lentivirus}

Small interfering RNAs targeting the mouse IL-1 $\beta$ gene were designed by the Shanghai GeneChem Co., Ltd., China. The optimal sequence of small interfering RNAs against mouse IL-1 $\beta$ (5'-TCAAAGGAAAGAATCTATA-3') was then cloned into the plasmid pGCL-GFP, which encodes a human immunodeficiency virus (HIV)-derived lentiviral vector containing a multiple cloning site for the insertion of shRNA constructs to be driven by an upstream U6 promoter and a downstream cytomegalovirus promoter-GFP (marker gene) cassette flanked by loxP sites. The NS shRNA was constructed by a similar process (5'-TTCTCCGAACGTGTCACGT-3'). These modified plasmids were further co-transfected into HEK293T cells with lentiviral packaging plasmids to generate an IL-1 $\beta$ shRNA-expressing lentivirus or a control shRNA-expressing lentivirus. HEK293T cells were plated in 6 -well plates $\left(6 \times 10^{5}\right.$ cells/well $)$ and cultured for $24 \mathrm{~h}$ before the transduction of lentiviral vectors (as shown in Fig. 1b). After 2 days of infection, the medium was replaced and the cells were further incubated until $48 \mathrm{~h}$ as required.

\section{Drugs and treatment}

LPS from Escherichia coli (0111: B4) was purchased from Sigma-Aldrich (St. Louis, MO, USA). The LPS injections were performed intraperitoneally in a $1 \mathrm{mg} / \mathrm{kg}$ injection volume. LPS solutions were prepared in saline $(0.9 \%$ $\mathrm{NaCl}$ ), and the control was performed intraperitoneally 


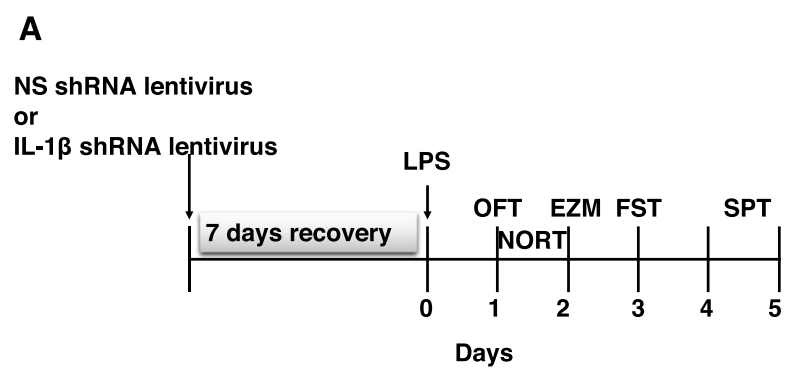

B

HEK293 cell
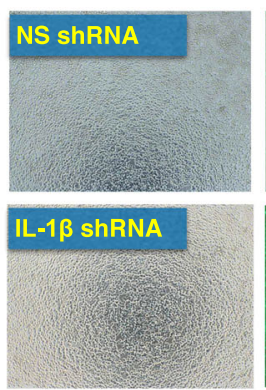

DG regions

C
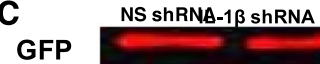

$\beta$-actin

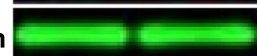

D

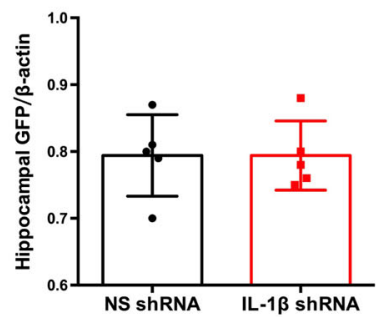

DG regions of hippocampus
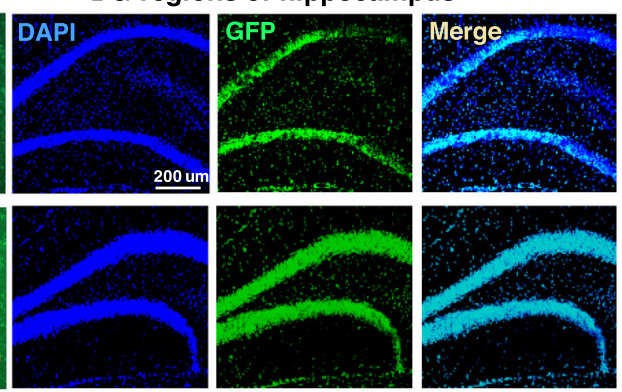

$D G$ regions

27 kDa

$45 \mathrm{kDa}$

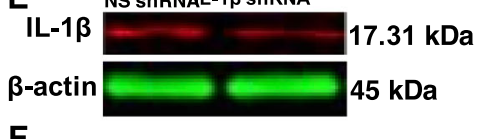

$F$

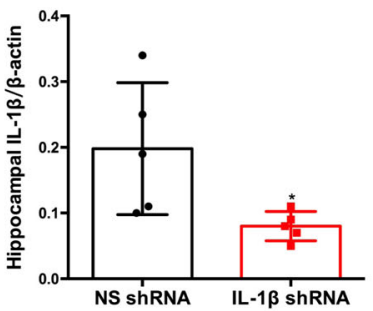

Fig. 1 Timeline of the experimental design and confirmation of the effectiveness of the IL-1 $\beta$ shRNA lentivirus in mice. a Experimental procedure for the test schedule. NS shRNA or IL-1 $\beta$ shRNA were microinfused into bilateral DG regions of the hippocampus of mice, followed by a 7-day recovery. LPS (1 mg/kg, i.p.) or its vehicle was administered 7 days after the viral infusions (day 0), and then, $24 \mathrm{~h}$ later, the OFT, NORT, EZM, FST, and SPT were conducted. $\mathbf{b}$ NS shRNA or IL-1 $\beta$ shRNA were well expressed in the HEK293 cells and at the hippocampal microinjection sites in the DG regions of the hippocampus, as indicated by GFP (green) under fluorescence microscopy. Scale bars = $100 \mu \mathrm{m}$ (HEK293 cell) or $200 \mu \mathrm{m}$ (hippocampus). $\mathbf{c}-\mathbf{f}$ The expressions of GFP $(\mathbf{c}, \mathbf{d})$ and IL-1 $(\mathbf{e}, \mathbf{f})$ in the DG regions of the hippocampus were shown and normalized by the level of $\beta$-actin. The data are expressed as the mean \pm S.E.M $\left(n=3\right.$ per group for western blotting). ${ }^{*} p<0.05$ compared with the NS shRNA group

with saline at $10 \mathrm{ml} / \mathrm{kg}$. In addition, malondialdehyde (MDA), superoxide dismutase (SOD), and tumor necrosis factor (TNF- $\alpha$ ) test kits were obtained from Nanjing Jiancheng Bioengineering Institute (Nanjing, China). BDNF ELISA kits were obtained from Wuhan Boster Bioengineering Institute (Wuhan, China).

For stereotactic injection of IL- $1 \beta$ shRNA lentivirus ( $1 \mu \mathrm{l} /$ side $)$ or NS shRNA lentivirus $(1 \mu \mathrm{l} /$ side $)$ into the dentate gyrus (DG) regions of the hippocampus, the mice were anesthetized with pentobarbital sodium and placed on a stereotactic apparatus (RWD Life Science, Shenzhen, China). The injection was performed through drilled holes in the skull and was into the DG regions of the hippocampus using the following coordinates: $2.5 \mathrm{~mm}$ posterior, $\pm 2.5 \mathrm{~mm}$ lateral, and $-2.0 \mathrm{~mm}$ ventral from the bregma. The injection speed was set at $0.2 \mu \mathrm{l} / \mathrm{min}$, and the needle was left in place for $5 \mathrm{~min}$ following the injection. The mice were allowed to recover for 7 days and were handled every other day to reduce the stress associated with handling at the time of testing. The schedule of drug treatment and test orders was shown in Fig. 1a.

\section{Open field test (OFT)}

The OFT was performed in a $50 \times 50 \times 39 \mathrm{~cm}$ white plexiglass box; the box was divided into four identical 
squares. Line crossing (four paws placed into a new square) and rearing (with both front paws raised from the floor) were recorded for $5 \mathrm{~min}$ in a sound-controlled room. The apparatus was cleaned with $5 \%$ ethanol to remove scent clues after each test. The OFT was used to evaluate the locomotor activity in the mice.

\section{Novel object recognition test (NORT)}

The NORT was performed in a $50 \times 50 \times 39 \mathrm{~cm}$ white, plexiglass box. In the first day, the mice were subjected to a training session in which the mice were exposed to two of the same objects, and the exploration time was recorded with two stopwatches. Twenty-four hours later, the mice were placed back in the behavioral chamber, and one of the familiar objects was replaced by a novel object. The times spent exploring the familiar and the novel objects were recorded. The NORT was used to evaluate the spatial cognitive ability of the mice.

\section{Elevated zero maze (EZM)}

The EZM was a ring-shaped apparatus, elevated $40 \mathrm{~cm}$ from the floor. This apparatus consisted of a circular platform (outer diameter $46 \mathrm{~cm}$, width $5.5 \mathrm{~cm}$ ) divided into four quadrants of equal lengths with two open arms (with a 1-cm-high curb to prevent falls) and two equal closed arms (surrounded by a 20-cm wall from the surface of the maze). Each mouse was placed at the open arm of the EZM; 5 min of free exploration was recorded by a video camera, and the time spent in the open arm and total number of entries into the open arm during the test was measured, evaluated, and presented. EZM was used to evaluate the anxiety-like behavior in the mice.

\section{Forced swimming test (FST)}

The FST were performed in a glass cylindrical tank that was $60 \mathrm{~cm}$ high and $38 \mathrm{~cm}$ wide and was filled with fresh water $\left(23 \pm 2{ }^{\circ} \mathrm{C}\right)$ to the depth of $40 \mathrm{~cm}$. The total duration of immobility was recorded during the last 4 min of a 6-min time frame. The duration of immobility was considered as the time when the mouse made only the small movements necessary to keep the head above water. The FST was used to evaluate the depression-like behavior in the mice.

\section{Sucrose preference test (SPT)}

The mice were habituated to $1 \%$ sucrose for $24 \mathrm{~h}$, and the bottle size was consistent across animals. On testing day, the mice were water-deprived for $6 \mathrm{~h}$ and then presented with pre-weighed identical bottles of $1 \%$ sucrose and water. The bottles were removed $12 \mathrm{~h}$ later and weighed to determine the consumption of each fluid. SPT also evaluated the depression-like behavior in the mice.
Enzyme-linked immunosorbent assay (ELISA)

Concentrations of MDA, SOD, TNF- $\alpha$, and BDNF were quantified using ELISA kits according to the manufacturer's protocol. Hippocampal tissues were homogenized in PBS (Solarbio, Beijing, China) at a ratio of 1:9 (weight to volume) in a glass homogenizer. Supernatant protein concentrations were determined after centrifugation at $12,000 \mathrm{rpm}$ for $10 \mathrm{~min}$ at $4{ }^{\circ} \mathrm{C}$. For each sample, $200 \mu \mathrm{l}$ of extracted protein was used for detection. The absorbance was read on a spectrophotometer at a wavelength of $450 \mathrm{~nm}$. The concentrations of each parameter were calculated according to the standard curve.

\section{Immunoblotting}

Briefly, hippocampal tissues were homogenized in a radioimmunoprecipitation assay (RIPA) lysis buffer (Beyotime, Beijing, China) containing protease inhibitors (Promega, Madison, USA) and phosphatase inhibitors (Sangon, Shanghai, China) and then centrifuged at 12,000 rpm for $30 \mathrm{~min}$ at $4{ }^{\circ} \mathrm{C}$. The supernatant was harvested and quantified by the BCA Protein Assay Kit (Beyotime, Beijing, China). Samples (20 $\mu \mathrm{g}$ of protein in each lane) were separated using 10\% SDS-PAGE gels and subsequently transferred to PVDF membranes $(0.22 \mu \mathrm{m}$; Millipore, Temecula, CA, USA). The samples were then incubated overnight with anti-IL-1 $\beta$ (1:1000; Cell Signaling, Danvers, MA, USA), anti-nuclear factor erythroid-derived 2-like 2 (Nrf2) (1:1000; Abcam, Cambridge, MA, USA), rabbit anti-heme oxygenase 1 (HO1) (1:1000; Millipore, Temecula, CA, USA), or rabbit anti-VGF (1:1000; Abcam, Cambridge, MA, USA) primary antibody at $4{ }^{\circ} \mathrm{C}$. Afterward, the membranes were incubated with horseradish peroxidase-conjugated goat anti- $\beta$-actin secondary antibodies (1:1000; Cell Signaling Technology, MA, USA) for $60 \mathrm{~min}$. The detection and quantification of specific bands were performed using Image software (NIH, Bethesda, MD, USA).

\section{Immunohistochemistry}

The mice were perfused with $60 \mathrm{ml}$ of saline and $30 \mathrm{ml}$ of $4 \%$ paraformaldehyde (PFA). The brain was taken out and post-fixed with same fixative overnight, and then, the brain was treated with $30 \%$ sucrose at $4{ }^{\circ} \mathrm{C}$ for 2 days. Brain tissue was sectioned with a thickness of $20 \mu \mathrm{m}$ and pasted into microscope slides. Brain sections were blocked with blocking buffer (BSA, PBS with $0.2 \%$ Triton $\mathrm{X}-100)$ for $1 \mathrm{~h}$. The sections were incubated overnight with anti-VGF primary antibody or anti-IL-1 $\beta$ primary antibody at $4{ }^{\circ} \mathrm{C}$. The sections were washed in PBS and incubated with fluorescent secondary antibodies, the donkey anti-rabbit-conjugated AlexaFluor 594-labeled and goat anti-rabbit secondary antibodies (1:1000; Invitrogen), for $1 \mathrm{~h}$ at room temperature. DNA (nuclei) was stained with DAPI (Solarbio, Beijing, China) for $15 \mathrm{~min}$, 
mounted onto slides and coverslips with ProLong mounting medium (Invitrogen). The images were captured using the Olympus system and analyzed with ImageJ software.

\section{Data analysis}

Data are expressed as the mean \pm standard error of the mean (S.E.M.). Data were analyzed by a two-way ANOVA followed by Newman-Keuls post hoc test using the GraphPad Prism software (Version 6.0, Prism software for PC, GraphPad). The criterion for significance was $p<0.05$.

\section{Results}

Inhibition of IL- $1 \beta$ activity produced by IL-1 $\beta$ shRNA blocked the cognitive dysfunction and the anxiety- and depression-like behaviors induced by LPS in mice

NS shRNA or IL-1 $\beta$ shRNA were well expressed in HEK293 cells and at the hippocampal microinjection sites in the DG regions, as indicated by GFP (green) under fluorescence microscopy (as shown in Fig. 1b). The expression of GFP in the DG regions of the hippocampus did not show significant differences between the NS shRNA and IL- $1 \beta$ shRNA-treated mice $(p>0.05$, Fig. 1c, d). However, IL-1 $\beta$ shRNA lentivirus microinjection into the DG regions of the hippocampus significantly decreased the expression of IL-1 $\beta$ in the DG regions of the hippocampus compared with the NS shRNA lentivirus treatment, indicating the effectiveness of this lentivirus (as shown in Fig. 1e, f).

To investigate the role of IL- $1 \beta$ in cognitive dysfunction and in the anxiety- and depression-like behaviors induced by LPS in mice, the mice were treated with either IL-1 $\beta$ shRNA lentivirus or NS shRNA lentivirus by microinjection into the DG regions of the hippocampus. After 7 days of recovery, LPS (1 mg/kg, i.p.) or saline were administered. Then, the OFT (Fig. 2a, b) was conducted $24 \mathrm{~h}$ after the injection of LPS. The two-way ANOVA revealed significant differences for the LPS treatment [line crossings: $F(1,60)=51.16 ; p<0.0001$; rearings: $F(1,60)=62.08 ; p<0.0001]$, the IL-1 $\beta$ shRNA treatment [line crossings: $F(1,60)=10.08 ; p=0.0024$; rearings: $F(1,60)=24.76 ; p<0.0001]$ and the LPS treatment $\times$ IL-1 $\beta$ shRNA interaction [line crossings: $F$ $(1,60)=22.48 ; p<0.0001$; rearings: $F(1,60)=13.17$; $p=0.0006$ ] on line crossings (Fig. 2a) and rearings (Fig. 2b). Post hoc analysis showed that the decrease in line crossings $(p<0.01)$ and rearings $(p<0.01)$ produced by LPS was completely prevented by treating the animals with the IL- $1 \beta$ shRNA (both $p<0.01$ ).

Additionally, the changes in cognitive ability in the mice were evaluated by NORT. Figure 2c showed that the administration of LPS alone or in combination with IL-1 $\beta$ shRNA did not modify the recognition index for familiar objects of mice in the training session of NORT
(LPS treatment $[F(1,60)=0.1210 ; p=0.7292]$, IL-1 $\beta$ shRNA treatment $[F(1,60)=0.0018 ; p=0.9664]$, LPS treatment $\times$ IL- $1 \beta$ shRNA interaction $[F(1,60)=0.0070$; $p=0.9336])$. However, the recognition index for novel objects was significantly changed by LPS alone or in combination with IL-1 $\beta$ shRNA treatment in mice (LPS treatment $[F(1,60)=26.43 ; p<0.0001]$, IL-1 $\beta$ shRNA treatment $[F(1,60)=12.17 ; p=0.0009]$, LPS treatment $\times$ IL-1 $\beta$ shRNA interaction $[F(1,60)=8.906 ; p=0.0041])$. Post hoc analysis shown that the decrease in recognition index $(p<0.01)$ produced by LPS was completely prevented by treating the animals with the IL-1 $\beta$ shRNA lentivirus $(p<0.01)$.

The anxiety-like behaviors were examined by EZM in mice. As shown in Fig. 2e, f, the duration in the open arms (LPS treatment $[F(1,60)=53.29 ; p<0.0001]$, IL$1 \beta$ shRNA treatment $[F(1,60)=8.717 ; p=0.0045]$, LPS treatment $\times$ IL-1 $\beta$ shRNA interaction $[F(1,60)=21.29$; $p<0.0001])$ and entries into the open arms (LPS treatment $[F(1,60)=54.68 ; p<0.0001]$, IL-1 $\beta$ shRNA treatment $[F(1,60)=4.199 ; p=0.0448]$, LPS treatment $\times$ IL-1 $\beta$ shRNA interaction $[F(1,60)=10.87 ; p=0.0016])$ were significantly changed by LPS and/or IL-1 $\beta$ shRNA treatment in mice. Post hoc analysis showed that the decreases in duration in the open arms $(p<0.01)$ and in entries into the open arms $(p<0.01)$ produced by LPS were completely prevented by treating the animals with the IL-1 $\beta$ shRNA lentivirus $(p<0.01$ and $p<0.01$, respectively). Moreover, we also used FST and SPT to demonstrate the depression-like behaviors produced by the LPS alone or in combination with the IL- $1 \beta$ shRNA in mice. As shown in Fig. $2 g$, h, the immobility time and sucrose consumption were significantly changed by LPS alone [immobility: $F(1,60)=36.37 ; p<0.0001$, Fig. 2g; sucrose consumption: $F(1,60)=38.17 ; p<0.0001$, Fig. 2h], IL-1 $\beta$ shRNA [immobility: $F(1,60)=13.75$; $p=0.0005$, Fig. 2g; sucrose consumption: $F(1$, $60)=31.52 ; p<0.0001$, Fig, $2 \mathrm{~h}$ ], or in combination with IL-1 $\beta$ shRNA [immobility: $F(1,60)=12.03 ; p=0.0010$, Fig. 2g; sucrose consumption: $F(1,60)=16.08$; $p=0.0002$, Fig. 2h] in mice. Post hoc analysis showed that the increase in immobility time $(p<0.01)$ and the decrease in sucrose consumption $(p<0.05)$ produced by LPS were completely prevented by treating the animals with the IL- $1 \beta$ shRNA lentivirus $(p<0.01$ and $p<0.01$, respectively) in mice.

Inhibition of IL-1 $\beta$ activity mediated by IL-1 $\beta$ shRNA lentivirus alleviated the LPS-induced oxidative response in the hippocampus of mice

As shown in Fig. 3, the two-way ANOVA revealed significant effects of the LPS treatment [MDA: $F(1,20)=41.86$, $p<0.0001$, Fig. 3a; SOD: $F(1,20)=18.85, p=0.0003$, Fig. 3b; Nrf2: $F(1,16)=45.68, p<0.0001$, Fig. 3c, d; and 

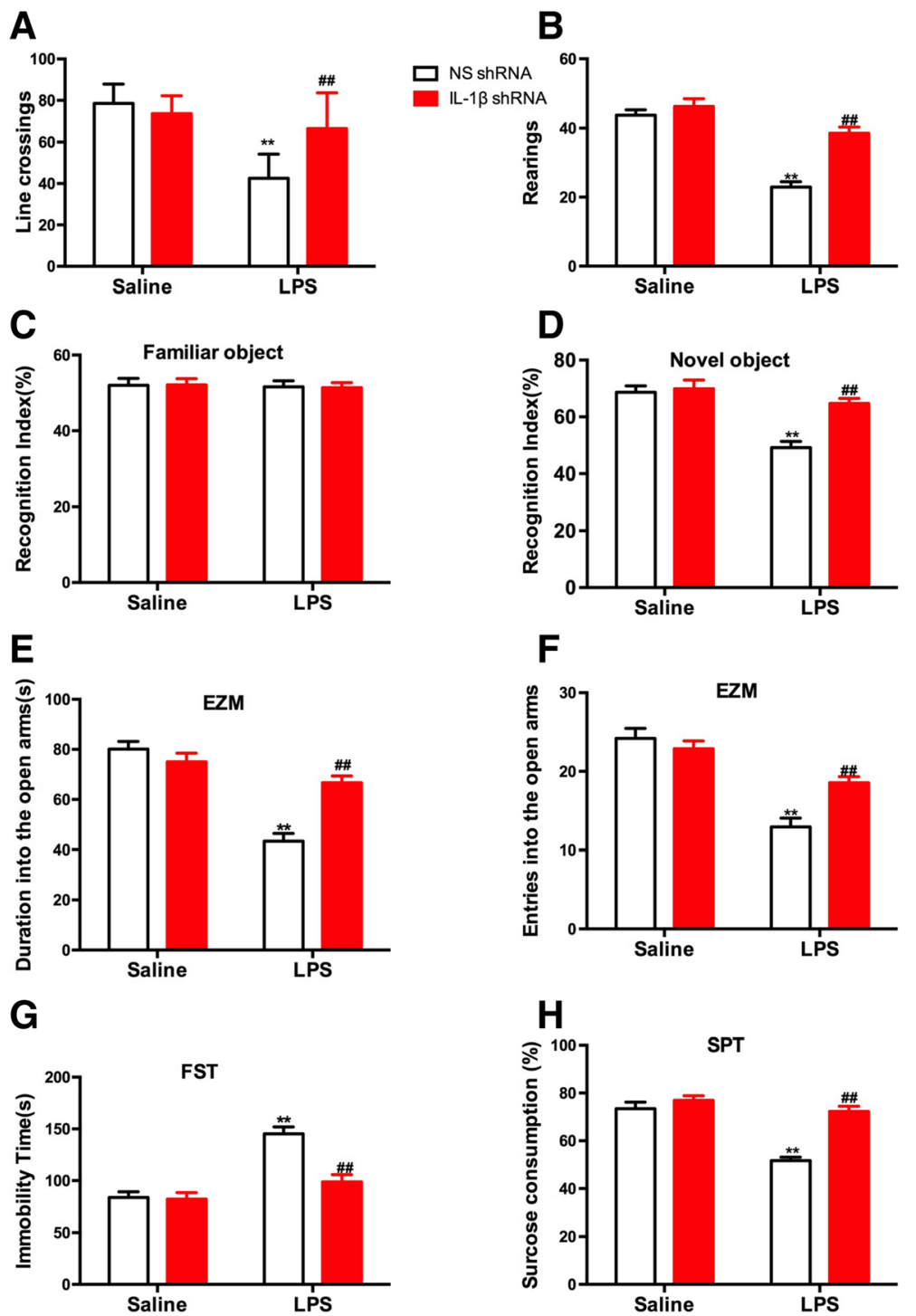

Fig. 2 The influence of IL-1 $\beta$ knock-down in the DG regions of the hippocampus on locomotor activity and on anxiety- and depressionlike behaviors induced by LPS in mice. Knock-down of IL-1 $\beta$ in the DG regions of the hippocampus alleviated the downregulation of locomotor activity induced by LPS, reflected by the line crossing (a) and rearing (b) in mice. c The recognition index had no significant difference for familiar objects among all the treatments in mice. $\mathbf{d}$ However, knock-down of IL-1 $\beta$ in the hippocampus alleviated the downregulation in the recognition index for novel objects induced by LPS in mice. The decrease $\mathbf{e}$ in duration in the open arms and $\mathbf{f}$ in entries into the open arms induced by LPS was blocked by pretreatment with the IL-1ß shRNA lentivirus in the DG regions of the hippocampus of mice. $\mathbf{g}$ The increase in immobility time in the FST induced by LPS was significantly alleviated by pretreatment with the IL-1 $\beta$ shRNA lentivirus into the DG regions of the hippocampus of mice. $\mathbf{h}$ The decrease of sucrose consumption in the SPT induced by LPS was blocked by pretreatment with the IL-1 $\beta$ shRNA lentivirus into the DG regions of the hippocampus of mice. The data are expressed as the mean \pm S.E.M ( $n=16$ per group). ${ }^{* *} p<0.01$ compared with the NS shRNA plus saline group; \#\#p $<0.01$ compared with NS shRNA + LPS group

HO1: $F(1,16)=41.70, p<0.0001$, Fig. 3e, f], the IL-1 $\beta$ shRNA treatment [MDA: $F(1,20)=2.683, p=0.0356$; SOD: $F(1,20)=5.151, p=0.0344 ; \operatorname{Nrf2:} F(1,16)=34.46$, $p<0.0001$; and HO1: $F(1,16)=23.96, p=0.0002]$, and the LPS treatment $\times$ IL- $1 \beta$ shRNA interaction [MDA: $F$ $(1,20)=6.352, p=0.0203$; SOD: $F(1,20)=7.235$, $p=0.0141 ; \mathrm{Nrf} 2: F(1,16)=17.39, p=0.0007$; and HO1:
$F(1,16)=27.83, p<0.0001]$ on the levels of MDA, SOD, $\mathrm{Nrf2}$, and HO1 in the hippocampus of mice. Post hoc analysis showed that the upregulation of MDA $(p<0.01$, Fig. 3a) and the downregulation of SOD $(p<0.05)$, Nrf2 $(p<0.01)$, and HO1 $(p<0.01)$ induced by LPS were completely prevented by treating the animals with the IL- $1 \beta$ shRNA lentivirus in the hippocampus. 
A

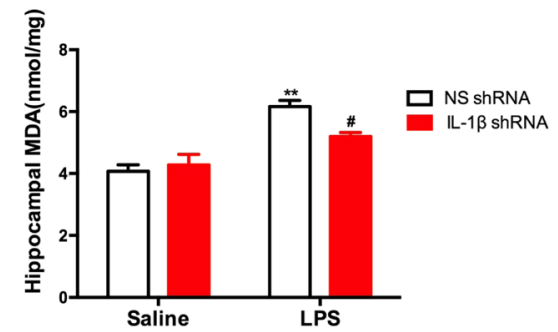

C

D
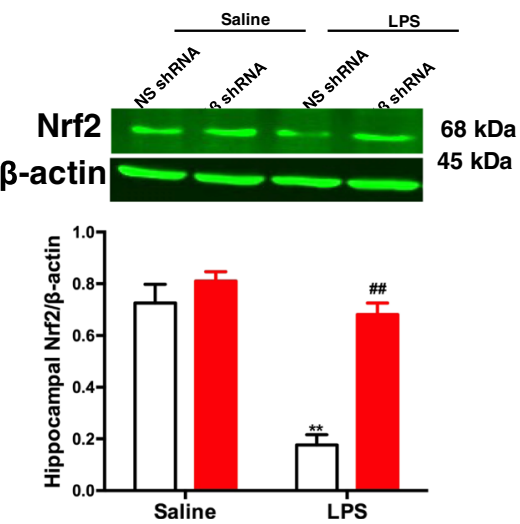

B

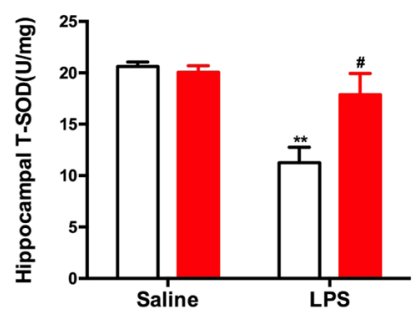

$\mathbf{E}$

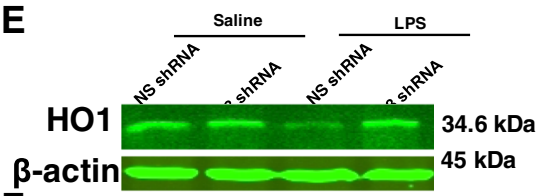

$\mathbf{F}$

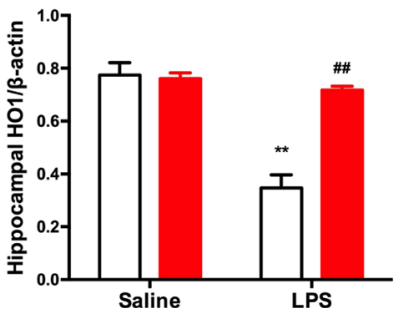

Fig. 3 The influence of pretreatment with IL-1 $\beta$ shRNA lentivirus on the levels of oxidative or anti-oxidative parameters induced by LPS in the hippocampus of mice. a The upregulation of MDA in the hippocampus induced by LPS was significantly alleviated by IL-1 $\beta$ knock-down in the mice. $\mathbf{b}$ The downregulation of SOD in the hippocampus induced by LPS was significantly alleviated by IL-1 $\beta$ knock-down in the mice. $\mathbf{c}$, e Representative immunoblots of Nrf2 and $\mathrm{HO} 1$ detected by western blotting with tissues from the hippocampus, and the rest of the panels are quantifications of the immunoblot bands of $\mathrm{Nrf2}$ (d) and HO1 (f). The decrease in Nrf2 and HO1 in the hippocampus induced by LPS was significantly alleviated by IL-1 $\beta$ knock-down. The data are expressed as the mean \pm S.E.M ( $n=3$ per group for western blotting and $n=6$ per group for ELISA). ${ }^{* *} p<0.01$ compared with the NS shRNA plus saline group; \#p $<0.05$ or \#\#p $<0.01$ compared with the NS shRNA + LPS group

Inhibition of IL-1 $\beta$ activity by IL-1 $\beta$ shRNA blocked LPSinduced pro-inflammatory cytokines in the hippocampus of mice

As shown in Fig. 4a, b, the IL-1 $\beta$-labeled positive cells in the DG regions of the hippocampus were significantly changed by the LPS treatment $[F(1,20)=99.18$; $p<0.0001]$, IL-1 $\beta$ shRNA treatment $[F(1,20)=32.65$; $p<0.0001]$ and the LPS treatment $\times$ IL- $1 \beta$ shRNA interaction $[F(1,20)=5.717 ; p=0.0267]$. Post hoc analysis showed that the increase of IL- $1 \beta$ ( $p<0.01$, Fig. 4b) induced by LPS was completely prevented by treating the animals with the IL- $1 \beta$ shRNA $(p<0.01)$. Additionally, the protein expression of IL-1 $\beta$ in the DG regions of the hippocampus was significantly changed by the LPS treatment $[F(1,16)=149.6 ; p<0.0001]$, the IL-1 $\beta$ shRNA treatment $[F(1,16)=110.1 ; p<0.0001]$, and the LPS treatment $\times$ IL-1 $\beta$ shRNA interaction $[F(1,16)=27.16$; $p<0.0001]$. Post hoc analysis showed that the increase in IL- $1 \beta$ ( $p<0.01$, Fig. $4 \mathrm{c}, \mathrm{d})$ induced by LPS was completely prevented by treating the animals with the IL- $1 \beta$ shRNA $(p<0.01)$. Furthermore, the level of TNF- $\alpha$ in the hippocampus was significantly changed by the LPS treatment $[F(1,20)=121.1 ; p<0.0001]$, the IL-1 $\beta$ shRNA treatment $[F(1,20)=41.03 ; p<0.0001]$, and the LPS treatment $\times$ IL- $1 \beta$ shRNA interaction $[F(1,20)=20.91$; $p=0.0002]$. Post hoc analysis showed that the increase in TNF- $\alpha$ ( $p<0.01$, Fig. 4e) induced by LPS was completely prevented by treating the animals with the IL-1 $\beta$-shRNA lentivirus.

\section{Inhibition of IL-1 $\beta$ activity by IL-1 $\beta$ shRNA lentivirus blocked the downregulation of VGF and BDNF by LPS in the hippocampus of mice}

Finally, we examined the expressions of VGF (Fig. 5a-d) and BDNF (Fig. 5e) in the hippocampus of mice. The fluorescent expression (Fig. 5a, b) and protein expression (Fig. 5c, d) of VGF were significantly changed by the LPS treatment [fluorescent expression: $F(1,20)=14.66$; $p=0.0010$; protein expression: $F(1,16)=55.21$; $p<0.0001$ ], the IL-1 $\beta$ shRNA treatment [fluorescent expression: $F(1,20)=10.57 ; p=0.0040$; protein expression: $F(1,16)=13.00 ; p=0.0024 \mathrm{l}$, and the LPS treatment $\times$ IL-1 $\beta$ shRNA interaction [fluorescent expression: $F(1,20)=4.493 ; p=0.0467$; protein expression $F(1,16)=27.84 ; p<0.0001]$. However, pretreatment with the IL-1 $\beta$ shRNA lentivirus significantly alleviated the downregulating effects of LPS (fluorescent expression: $p<0.01$; protein expression: $p<0.01$ ). In 


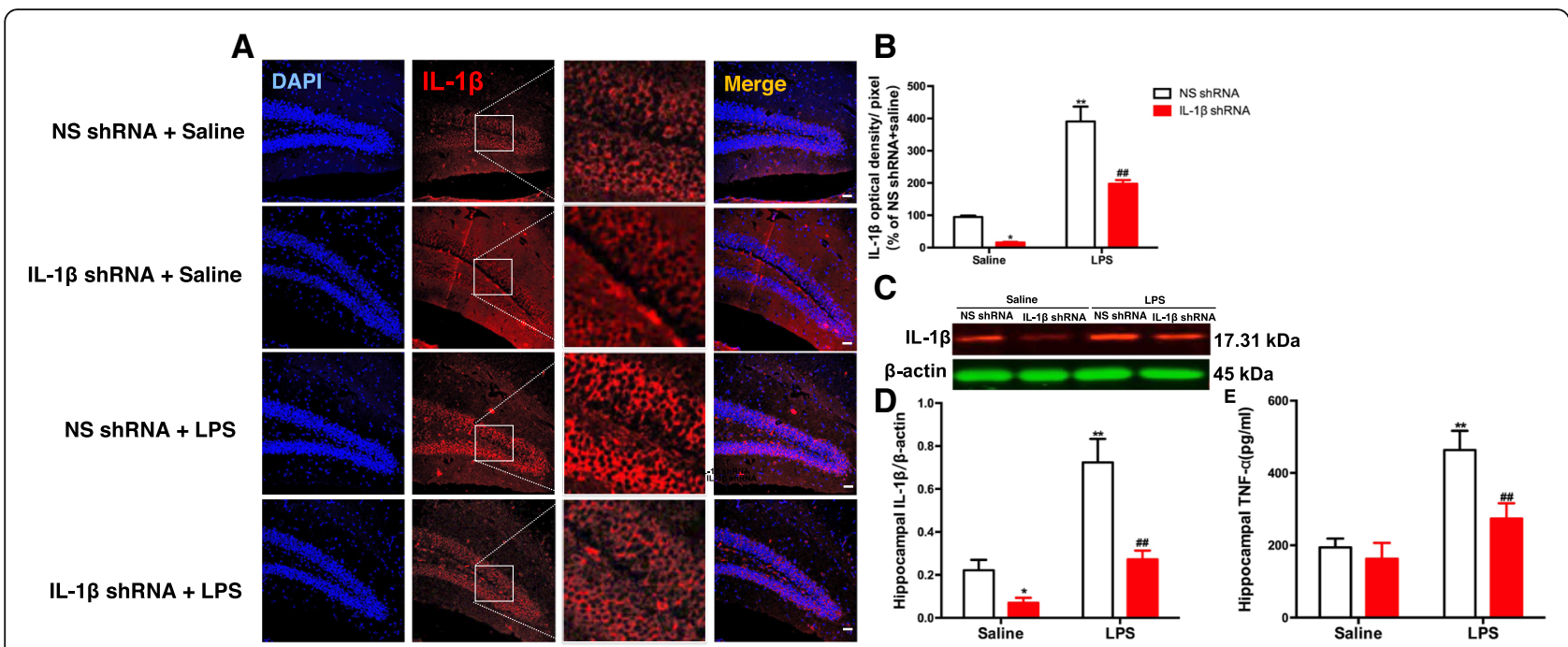

Fig. 4 The upregulation of neuroinflammatory factors in the hippocampus induced by LPS was blocked by pretreatment with IL-1 $\beta$ shRNA lentivirus in mice. a Mouse hippocampus samples were examined by using immunofluorescent analysis; sections were labeled with anti-IL-1 $\beta$ antibody (red) and with fluorescent nuclear DAPI staining (blue) and visualized using a fluorescence microscope. Scale bar $=50 \mu \mathrm{m}$. $\mathbf{b}$ Quantification of the IL-1 $\beta$ optical density per pixel was significantly decreased by IL-1 $\beta$ shRNA lentivirus and showed that the upregulation of IL-1 $\beta$ levels induced by LPS was significantly alleviated by the IL-1 $\beta$ shRNA lentivirus in mice. $\mathbf{c}$, $\mathbf{d}$ The hippocampal IL-1 $\beta$ protein expression was significantly decreased by LPS, and the IL-1 $\beta$ shRNA lentivirus alleviated this effect. $\mathbf{e}$ The upregulation of TNF-a in the hippocampus induced by LPS was significantly alleviated by pretreatment with IL-1 $\beta$ shRNA lentivirus in mice. The data are expressed as the mean \pm S.E.M $(n=3$ per group for western blotting and $n=6$ per group for immunofluorescent analysis or ELISA). ${ }^{*} p<0.05$ or ${ }^{* *} p<0.01$ compared with the NS shRNA plus saline group; \#\#p $<0.01$ compared with the NS shRNA + LPS group

addition, as shown in Fig. 5e, the two-way ANOVA revealed significant effects of the LPS treatment $[F$ (1, $20)=27.04 ; p<0.0001]$, the IL-1 $\beta$ shRNA treatment $[F$ $(1,20)=7.076 ; p=0.0150]$, and the LPS treatment $\times$ IL-1 $\beta$ shRNA interaction $[F(1,20)=12.26 ; p=0.0022]$ on the levels of BDNF in the hippocampus of mice. However, the knock-down of IL-1 $\beta$ in the DG regions of the hippocampus significantly blocked the effects of LPS $(p<0.01)$.

\section{Discussion}

A growing body of evidence suggests that activation of the immune response following systemic infection often results in neuroinflammatory responses and consequently induces neuropsychiatric symptoms in animal models and humans [7-10, 24-26]. Specifically, inflammation in the context of the nervous system, termed "neuroinflammation," has been reported in patients with neuropsychiatric disorders and is typically associated with microglial activation and the production of cytokines. Pro-inflammatory cytokines, including IL-1 $\beta$ and TNF- $\alpha$, are secreted primarily by the microglia [27-29]. A recent study also raised the possibility that cytokines might be used as endogenous biomarkers of the efficacy of antidepressants [30]. Notably, IL-1 $\beta$ has been proposed to be a key mediator in a variety of behavioral actions of neuropsychiatric disorders [31]. Once activated, IL- $1 \beta$ can activate immune-related cells, such as monocytes, to produce the corresponding immune effects, which in turn aggravate the inflammatory reaction [32]. Moreover, pharmacological inhibition of IL-1 $\beta$ signaling has been shown to be beneficial in some autoimmune and autoinflammatory diseases, making IL-1 $\beta$ a promising therapeutic target in neuroinflammatory conditions [11, 12, 33]. The first objective of our present study was to explore the involvement of IL- $1 \beta$ in the behavioral dysfunction induced by a peripheral immune challenge in mice. The mechanisms of central or peripheral treatment with LPS on behavioral dysfunctions in rodents trigger an increase in the expression of pro-inflammatory cytokines [7, 34, 35]. Herein, we found that peripheral administration of LPS causes the elevation of IL-1 $\beta$ and TNF- $\alpha$ levels in the hippocampus of mice, which may have contributed to the behavioral alterations. Additionally, our current data are consistent with previous evidence that indicates that neuroinflammation may play a crucial role in the pathophysiology of anxiety, major depression, and recognition memory deficits [36-38]. Interestingly, our present study investigated the significant alleviating effects of IL-1 $\beta$ knock-down in the DG regions of the hippocampus on the memory deficits and the depression- and anxiety-like behaviors, indicating the key role of IL- $1 \beta$ in the neuropsychiatric dysfunctions induced by LPS in mice.

In addition to the neuroinflammation induced by LPS administration in rodents, oxidative stress may also play an important role in neuropsychiatric disorders. Our current observations further confirmed that the levels of 


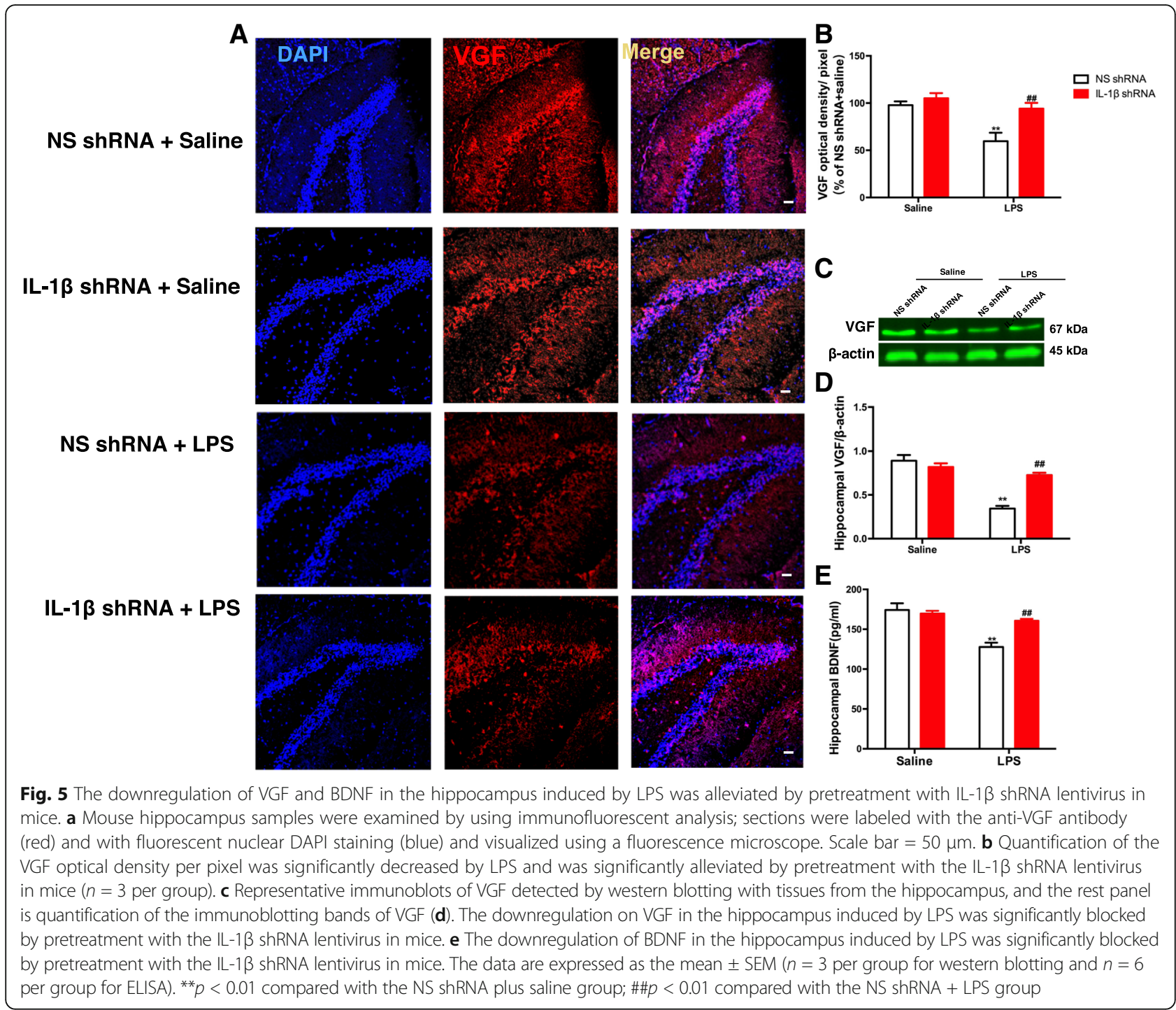

markers reflecting the status of oxidative stress, such as MDA, were significantly altered by LPS administration. However, reduced SOD, Nrf2, and HO1 levels by LPS were also found in our present study, indicating that reversion of the harmful changes in oxidative stress markers would be beneficial for neuroinflammation-induced neuropsychiatric disorder therapy. Nrf2, a transcription factor involved in the cellular defense against oxidative stress and the neuroinflammatory response, is one potential target of interest for the treatment of neuropsychiatric disorders [39-41]. In addition, the cytoprotective enzyme $\mathrm{HO} 1$ is an Nrf2 target protein and HO1 upregulation contributes to the antioxidant and anti-inflammatory properties [42, 43]. However, very few studies have assessed whether SOD, Nrf2, and HO1 were downregulated by IL-1 $\beta$ in the neuropsychiatric dysfunctions induced by LPS in mice. Herein, one of the most striking findings of our current study was that IL-1 $\beta$ knock-down in the hippocampus of mice significantly antagonized the LPS-induced decrease in SOD, Nrf2, and HO1. Thus, neuroinflammation, along with oxidative stress, perpetuates a vicious cycle that ultimately leads to the behavioral alterations associated with neuropsychiatric disorders.

BDNF and VGF are involved in many aspects of neuronal functioning including synaptic plasticity, neurogenesis, and neuronal survival and are associated with core features of neuropsychiatric disorders [17, 44-46]. Concerning our results, LPS administration was associated with decreased BDNF and VGF levels in the brains of mice, which is in agreement with previous studies that have found that cytokines were increased in neuropsychiatric patients [36], which then led to neurotoxic effects by proinflammatory cytokines and oxidative stress-associated mechanisms that ultimately impaired BDNF and VGF expression $[7,47,48]$. Specifically, our data show that IL-1 $\beta$ knock-down alleviates the downregulation of BDNF and 
VGF in the hippocampus of LPS-treated mice. Our current data may further demonstrate the key role of IL- $1 \beta$ in the regulation of BDNF and VGF after LPS treatment in mice. Our results are consistent with a recent report that demonstrated that the pharmacological inhibition of IL-1 $\beta$ prevented depression-like behaviors [49], indicating that IL-1 $\beta$ is a key component of the inflammatory response of the brain and mediates the effect of inflammation on depression-like behaviors in mice. However, IL-1 $\beta$, acting via poorly understood mechanisms, appears to be a key cytokine in causing decreases in BDNF and VGF along with presumably related behavioral dysfunctions induced by LPS in mice. Recent studies demonstrated that IL-1 $\beta$ suppressed TrkB-mediated BDNF signaling and the cAMP response element-binding protein (CREB) [50], a transcription factor regulating BDNF and VGF expression $[51,52]$. These findings may explain the mechanism of the involvement of IL-1 $\beta$ in the LPS-induced downregulation of BDNF and VGF in mice, suggest that IL- $1 \beta$ is critical for the development of depression-like behaviors, and highlight IL-1 $\beta$ as a potential novel therapeutic target for the treatment of mood disorders.

\section{Conclusion}

Taken together, alterations in the pro-inflammatory cytokines and oxidative stress parameters may contribute to the molecular basis of memory deficits and anxiety- and depression-like behaviors. To our knowledge, our present study is the first to report that IL- $1 \beta$ knock-down protects neuroinflammatory and oxidative responses, leading to VGF and BDNF activation in the hippocampus of mice. Our current study may provide novel insights into the development of new therapeutic approaches for neuropsychiatric disorders.

\begin{abstract}
Abbreviations
BDNF: Brain-derived neurotrophic factor; CREB: CAMP response elementbinding protein; DG: Dentate gyrus; ELISA: Enzyme-linked immunosorbent assay; EZM: Elevated zero maze; FST: Forced swimming test; HO1: Heme oxygenase 1; ICR: Imprinting control region; IL-1 $\beta$ : Interleukin-1 $\beta$; LPS: Lipopolysaccharide; MDA: Malondialdehyde; NORT: Novel object recognition test; Nrf2: Nuclear factor erythroid-derived 2-like 2; OFT: Open field test; RIPA: Radioimmunoprecipitation assay; SOD: Superoxide dismutase; SPT: Sucrose preference test; TNF-a: Tumor necrosis factor-a
\end{abstract}

\section{Acknowledgements}

We would like to thank Xin Zhao for the expert technical assistance.

\section{Funding}

This research was supported by the National Natural Science Foundation of China (No. 81671337; No. 81201050), Natural Science Foundation of Zhejiang Province (No. LQ12H09001), Natural Science Foundation of Ningbo (No. 2012A610251; No. 2017A610212), Ningbo municipal innovation team of life science and health (2015C110026), Xinmiao Talents Program of Zhejiang (2017R405030), and Student Research and Innovation Program (SIRP). This project is also sponsored by K.C. Wong Magna funded in Ningbo University and Li Dak Sum Yip Yio Chin Kenneth Li Marine Biopharmaceutical Development Fund, National 111 Project of China.

\section{Availability of data and materials}

Please contact author for data requests.

\section{Authors' contributions}

CW designed the research. ML, CL, HY, XC, XS, XS, JW, and YZ performed the research. $\mathrm{ML}, \mathrm{CL}, \mathrm{HY}, \mathrm{XC}, \mathrm{XS}, \mathrm{XS}, \mathrm{JW}, \mathrm{YZ}$, and $\mathrm{CW}$ were responsible of the design of the study and the interpretation of the results. $C L, M L$, and $C W$ conducted the statistical analysis and the draft of the manuscript. All authors read and approved the final manuscript.

\section{Ethics approval}

All animal experiments were approved by the Experimental Animal Ethics Committee of Ningbo University.

\section{Consent for publication}

Not applicable.

\section{Competing interests}

The authors declare that they have no competing interests.

\section{Publisher's Note}

Springer Nature remains neutral with regard to jurisdictional claims in published maps and institutional affiliations.

\section{Author details}

${ }^{1}$ Ningbo Key Laboratory of Behavioral Neuroscience, Ningbo University School of Medicine, 818 Fenghua Road, Ningbo, Zhejiang 315211, People's Republic of China. 'Zhejiang Provincial Key Laboratory of Pathophysiology, Ningbo University School of Medicine, 818 Fenghua Road, Ningbo, Zhejiang 315211, People's Republic of China. ${ }^{3}$ Department of Physiology and Pharmacology, Ningbo University School of Medicine, 818 Fenghua Road, Ningbo, Zhejiang 315211, People's Republic of China. ${ }^{4}$ Li Dak Sum Yip Yio Chin Kenneth Li Marine Biopharmaceutical Research Center, Ningbo University, Ningbo 315211, People's Republic of China.

Received: 7 June 2017 Accepted: 14 September 2017

Published online: 20 September 2017

\section{References}

1. Nazem A, Sankowski R, Bacher M, Al-Abed Y. Rodent models of neuroinflammation for Alzheimer's disease. J Neuroinflammation. 2015;12:74.

2. Badshah H, Ali T, Kim MO. Osmotin attenuates LPS-induced neuroinflammation and memory impairments via the TLR4/NFKB signaling pathway. Sci Rep. 2016;6:24493.

3. Faroog RK, Asghar K, Kanwal S, Zulqernain A. Role of inflammatory cytokines in depression: focus on interleukin-1 $\beta$. Biomed Rep. 2017;6:15-20.

4. Yue N, Huang H, Zhu X, Han Q, Wang Y, Li B, Liu Q, Wu G, Zhang Y, Yu J. Activation of P2X7 receptor and NLRP3 inflammasome assembly in hippocampal glial cells mediates chronic stress-induced depressive-like behaviors. J Neuroinflammation. 2017;14:102.

5. Deyama S, Ishikawa Y, Yoshikawa K, Shimoda K, Ide S, Satoh M, Minami M. Resolvin D1 and D2 reverse lipopolysaccharide-induced depression-like behaviors through the mTORC1 signaling pathway Int J Neuropsychopharmacol 2017; doi: https:/doi.org/10.1093/ijnp/pyx023.

6. Zager A, Brandão WN, Margatho RO, Peron JP, Tufik S, Andersen ML, Kornum BR, Palermo-Neto J. The wake-promoting drug Modafinil prevents motor impairment in sickness behavior induced by LPS in mice: role for dopaminergic D1 receptor. Prog Neuro-Psychopharmacol Biol Psychiatry. 2017. doi:10.1016/j.pnpbp.2017.05.003

7. Guo J, Lin P, Zhao X, Zhang J, Wei X, Wang Q, Wang C. Etazolate abrogates the lipopolysaccharide (LPS)-induced downregulation of the CAMP/pCREB/ BDNF signaling, neuroinflammatory response and depressive-like behavior in mice. Neuroscience. 2014;263:1-14.

8. Liang $\mathrm{R}$, Yong $\mathrm{S}$, Huang $\mathrm{X}$, Kong $\mathrm{H}, \mathrm{Hu} \mathrm{G}$, Fan Y. Aquaporin-4 mediates the suppressive effect of lipopolysaccharide on hippocampal neurogenesis. Neuroimmunomodulation. 2016;23:309-17.

9. Jangra A, Sriram CS, Lahkar M. Lipopolysaccharide-induced behavioral alterations are alleviated by sodium phenylbutyrate via attenuation of oxidative stress and neuroinflammatory cascade. Inflammation. 2016;39: $1441-52$. 
10. Davis RL, Stevens CW, Thomas CJ. The opioid antagonist, $\beta$-funaltrexamine inhibits lipopolysaccharide-induced neuroinflammation and reduces sickness behavior in mice. Physiol Behav. 2017;173:52-60.

11. Koo JW, Duman RS. Evidence for IL-1 receptor blockade as a therapeutic strategy for the treatment of depression. Curr Opin Investig Drugs. 2009;10:664-71.

12. Ben-Menachem-Zidon O, Ben-Menahem Y, Ben-Hur T, Yirmiya R. Intrahippocampal transplantation of neural precursor cells with transgenic overexpression of IL-1 receptor antagonist rescues memory and neurogenesis impairments in an Alzheimer's disease model. Neuropsychopharmacology. 2014;39:401-14

13. Song C, Horrobin D. Omega-3 fatty acid ethyl-eicosapentaenoate, but not soybean oil, attenuates memory impairment induced by central IL-1beta administration. J Lipid Res. 2004;45:1112-21.

14. Anisman H, Gibb J, Hayley S. Influence of continuous infusion of interleukin1 beta on depression-related processes in mice: corticosterone, circulating cytokines, brain monoamines, and cytokine mRNA expression. Psychopharmacology. 2008;199:231-44.

15. Goshen I, Yirmiya R. Interleukin-1 (IL-1): a central regulator of stress responses. Front Neuroendocrinol. 2009;30:30-45.

16. Thakker-Varia S, Jean YY, Parikh P, Sizer CF, Jernstedt Ayer J, Parikh A, Hyde TM, Buyske S, Alder J. The neuropeptide VGF is reduced in human bipolar postmortem brain and contributes to some of the behavioral and molecular effects of lithium. J Neurosci. 2010;30:9368-80.

17. Jiang C, Salton SR. The role of neurotrophins in major depressive disorder. Transl Neurosci. 2013;4:46-58.

18. Lin P, Wang C, Xu B, Gao S, Guo J, Zhao X, Huang H, Zhang J, Chen X, Wang Q, Zhou W. The VGF-derived peptide TLQP62 produces antidepressant-like effects in mice via the BDNF/TrkB/CREB signaling pathway. Pharmacol Biochem Behav. 2014;120:140-8.

19. Harrisberger F, Smieskova R, Schmidt A, Lenz C, Walter A, Wittfeld K, Grabe HJ, Lang UE, Fusar-Poli P, Borgwardt S. BDNF Val66Met polymorphism and hippocampal volume in neuropsychiatric disorders: a systematic review and meta-analysis. Neurosci Biobehav Rev. 2015;55:107-18.

20. Zheleznyakova GY, Cao H, Schiöth HB. BDNF DNA methylation changes as a biomarker of psychiatric disorders: literature review and open access database analysis. Behav Brain Funct. 2016;12:17.

21. Mariga A, Mitre M, Chao MV. Consequences of brain-derived neurotrophic factor withdrawal in CNS neurons and implications in disease. Neurobiol Dis. 2017:97:73-9.

22. Sulakhiya K, Kumar P, Jangra A, Dwivedi S, Hazarika NK, Baruah CC, Lahkar M. Honokiol abrogates lipopolysaccharide-induced depressive like behavior by impeding neuroinflammation and oxido-nitrosative stress in mice. Eur J Pharmacol. 2014;744:124-31.

23. Custódio CS, Mello BSF, Filho AJMC, de Carvalho Lima CN, Cordeiro RC, Miyajima F, Réus GZ, Vasconcelos SMM, Barichello T, Quevedo J, de Oliveira AC, de Lucena DF, Macedo DS. Neonatal immune challenge with lipopolysaccharide triggers long-lasting sex- and age-related behavioral and immune/neurotrophic alterations in mice: relevance to autism spectrum disorders. Mol Neurobiol. 2017; https://doi.org/10.1007/s12035-017-0616-1.

24. Isgren A, Sellgren C, Ekman CJ, Holmén-Larsson J, Blennow K, Zetterberg H, Jakobsson J, Landén M. Markers of neuroinflammation and neuronal injury in bipolar disorder: relation to prospective clinical outcomes. Brain Behav Immun. 2017. doi:10.1016/j.bbi.2017.05.002.

25. Müller N. Immunological aspects of the treatment of depression and schizophrenia. Dialogues Clin Neurosci. 2017;19:55-63.

26. Notter T, Coughlin JM, Gschwind T, Weber-Stadlbauer U, Wang Y, Kassiou M, Vernon AC, Benke D, Pomper MG, Sawa A, Meyer U. Translational evaluation of translocator protein as a marker of neuroinflammation in schizophrenia. Mol Psychiatry. 2017; https://doi.org/10.1038/mp.2016.248.

27. Mattei D, Djodari-Irani A, Hadar R, Pelz A, de Cossío LF, Goetz T, Matyash M, Kettenmann $\mathrm{H}$, Winter $\mathrm{C}$, Wolf SA. Minocycline rescues decrease in neurogenesis, increase in microglia cytokines and deficits in sensorimotor gating in an animal model of schizophrenia. Brain Behav Immun. 2014;38: $175-84$

28. Lu L, Li X, Xu P, Zheng Y, Wang X. Tenuigenin down-regulates the release of nitric oxide, matrix metalloproteinase- 9 and cytokines from lipopolysaccharide-stimulated microglia. Neurosci Lett. 2017:650:82-8.

29. Zhao H, Alam A, Chen Q, Eusman AM, Pal A, Eguchi S, Wu L, Ma D. The role of microglia in the pathobiology of neuropathic pain development: what do we know? Br J Anaesth. 2017;118:504-16.
30. Clarke M, Razmjou S, Prowse N, Dwyer Z, Litteljohn D, Pentz R, Anisman H, Hayley S. Ketamine modulates hippocampal neurogenesis and proinflammatory cytokines but not stressor induced neurochemical changes. Neuropharmacology. 2017;112:210-20.

31. Mendiola AS, Cardona AE. The IL-1 $\beta$ phenomena in neuroinflammatory diseases. J Neural Transm (Vienna). 2017; https://doi.org/10.1007/s00702017-1732-9.

32. Li Y, Liu L, Barger SW, Griffin WS. Interleukin-1 mediates pathological effects of microglia on tau phosphorylation and on synaptophysin synthesis in cortical neurons through a p38-MAPK pathway. J Neurosci. 2003:23:1605-11.

33. Volonté C, Apolloni S, Skaper SD, Burnstock G. P2X7 receptors: channels, pores and more. CNS Neurol Disord Drug Targets. 2012;11:705-21.

34. O'Connor JC, Lawson MA, André C, Moreau M, Lestage J, Castanon N, Kelley KW, Dantzer R. Lipopolysaccharide-induced depressive-like behavior is mediated by indoleamine 2,3-dioxygenase activation in mice. Mol Psychiatry. 2009;14:511-22

35. van Buel EM, Bosker FJ, van Drunen J, Strijker J, Douwenga W, Klein HC, Eisel UL. Electroconvulsive seizures (ECS) do not prevent LPSinduced behavioral alterations and microglial activation. J Neuroinflammation. 2015:12:232.

36. Carlos AJ, Tong L, Prieto GA, Cotman CW. IL-1 $\beta$ impairs retrograde flow of BDNF signaling by attenuating endosome trafficking. J Neuroinflammation. 2017;14:29.

37. Ito N, Hirose E, Ishida T, Hori A, Nagai T, Kobayashi Y, Kiyohara H, Oikawa T, Hanawa T, Odaguchi $\mathrm{H}$. Kososan, a Kampo medicine, prevents a social avoidance behavior and attenuates neuroinflammation in socially defeated mice. J Neuroinflammation. 2017;14:98.

38. Hendriksen E, van Bergeijk D, Oosting RS, Redegeld FA. Mast cells in neuroinflammation and brain disorders. Neurosci Biobehav Rev. 2017;79: 119-33.

39. Yao W, Zhang JC, Ishima T, Ren Q, Yang C, Dong C, Ma M, Saito A, Honda T, Hashimoto K. Antidepressant effects of TBE-31 and MCE-1, the novel Nrf2 activators, in an inflammation model of depression. Eur J Pharmacol. 2016; 793:21-7.

40. Chen J, Yin W, Tu Y, Wang S, Yang X, Chen Q, Zhang X, Han Y, Pi R. L-F001, a novel multifunctional ROCK inhibitor, suppresses neuroinflammation in vitro and in vivo: involvement of NF-kB inhibition and Nrf2 pathway activation. Eur J Pharmacol. 2017;806:1-9.

41. Wei CC, Kong YY, Li GQ, Guan YF, Wang P, Miao CY. Nicotinamide mononucleotide attenuates brain injury after intracerebral hemorrhage by activating Nrf2/HO-1 signaling pathway. Sci Rep. 2017;7:717.

42. Lei $X$, Lei L, Zhang Z, Cheng Y. Neuroprotective effects of lycopene pretreatment on transient global cerebral ischemia-reperfusion in rats: the role of the Nrf2/HO-1 signaling pathway. Mol Med Rep. 2016;13: 412-8.

43. Lee MH, Han MH, Lee DS, Park C, Hong SH, Kim GY, Hong SH, Song KS, Choi IW, Cha HJ, Choi YH. Morin exerts cytoprotective effects against oxidative stress in C2C12 myoblasts via the upregulation of Nrf2-dependent HO-1 expression and the activation of the ERK pathway. Int J Mol Med. 2017:39:399-406.

44. Thakker-Varia S, Alder J. Neuropeptides in depression: role of VGF. Behav Brain Res. 2009:197:262-78.

45. Leal G, Bramham CR, Duarte CB. BDNF and hippocampal synaptic plasticity. Vitam Horm. 2017:104:153-95.

46. Tanila H. The role of BDNF in Alzheimer's disease. Neurobiol Dis. 2017; 97:114-8

47. Ma M, Ren Q, Yang C, Zhang JC, Yao W, Dong C, Ohgi Y, Futamura T, Hashimoto K. Antidepressant effects of combination of brexpiprazole and fluoxetine on depression-like behavior and dendritic changes in mice after inflammation. Psychopharmacology. 2017;234:525-33.

48. Moraes MM, Galvão MC, Cabral D, Coelho CP, Queiroz-Hazarbassanov N, Martins MF, Bondan EF, Bernardi MM, Kirsten TB. Propentofylline prevents sickness behavior and depressive-like behavior induced by lipopolysaccharide in rats via neuroinflammatory pathway. PLoS One. 2017; 12:e0169446.

49. Li MX, Zheng HL, Luo Y, He JG, Wang W, Han J, Zhang L, Wang X, Ni L, Zhou HY, Hu ZL, Wu PF, Jin Y, Long LH, Zhang H, Hu G, Chen JG, Wang F. Gene deficiency and pharmacological inhibition of caspase-1 confers resilience to chronic social defeat stress via regulating the stability of surface AMPARs. Mol Psychiatry. 2017; https://doi.org/10.1038/mp.2017.76. 
50. Tong L, Prieto GA, Kramár EA, Smith ED, Cribbs DH, Lynch G, Cotman CW. Brain-derived neurotrophic factor-dependent synaptic plasticity is suppressed by interleukin-1 $\beta$ via p38 mitogen-activated protein kinase. J Neurosci. 2012;32(49):17714-24.

51. Rodríguez-Seoane C, Ramos A, Korth C, Requena JR. DISC1 regulates expression of the neurotrophin VGF through the PI3K/AKT/CREB pathway. J Neurochem. 2015 Nov;135(3):598-605.

52. Guo J, Lin $P$, Zhao X, Zhang J, Wei $X$, Wang $Q$, Wang C. Etazolate abrogates the lipopolysaccharide (LPS)-induced downregulation of the CAMP/pCREB/ BDNF signaling, neuroinflammatory response and depressive-like behavior in mice. Neuroscience. 2014 Mar 28:263:1-14.

Submit your next manuscript to BioMed Central and we will help you at every step:

- We accept pre-submission inquiries

- Our selector tool helps you to find the most relevant journal

- We provide round the clock customer support

- Convenient online submission

- Thorough peer review

- Inclusion in PubMed and all major indexing services

- Maximum visibility for your research

Submit your manuscript at www.biomedcentral.com/submit
Biomed Central 